\title{
Do patients with chronic unilateral orofacial pain due to a temporomandibular disorder show increased attending to somatosensory input at the painful side of the jaw?
}

\author{
Stefaan Van Damme \\ Charlotte Vanden Bulcke ${ }^{1}$, Linda Van den Berghe \\ Crombez ${ }^{1,4}$ \\ ${ }^{1}$ Department of Experimental-Clinical and Health Psychology, Ghent University, Ghent, Belgium \\ 2 Department of Dentistry, Ghent University Hospital, Ghent, Belgium \\ 3 Department of Movement and Sport Sciences, Ghent University, Ghent, Belgium \\ ${ }^{4}$ Center for Pain Research, University of Bath, Bath, United Kingdom \\ Corresponding Author: Stefaan Van Damme \\ Email address: stefaan.vandamme@ugent.be
} , Louise Poppe Geert

Background. Patients with chronic orofacial pain due to temporomandibular disorders (TMD) display alterations in somatosensory processing at the jaw, such as amplified perception of tactile stimuli, but the underlying mechanisms remain unclear. This study investigated one possible explanation, namely hypervigilance, and tested if TMD patients with unilateral pain showed increased attending to somatosensory input at the painful side of the jaw. Methods. TMD patients with chronic unilateral orofacial pain $(n=20)$ and matched healthy volunteers ( $n=20$ ) performed a temporal order judgment (TOJ) task indicated which one of two tactile stimuli, presented on each side of the jaw, they had perceived first. TOJ methodology allows examining spatial bias in somatosensory processing speed. Furthermore, after each block of trials, the participants rated the perceived intensity of tactile stimuli separately for both sides of the jaw. Finally, questionnaires assessing pain catastrophizing, fear-avoidance beliefs, and pain vigilance, were completed. Results. TMD patients tended to perceive tactile stimuli at the painful jaw side as occurring earlier in time than stimuli at the non-painful side but this effect did not reach conventional levels of significance $(p=.07)$. In the control group, tactile stimuli were perceived as occurring simultaneously. Secondary analyses indicated that the magnitude of spatial bias in the TMD group is positively associated with the extent of fear-avoidance beliefs. Overall, intensity ratings of tactile stimuli were significantly higher in the TMD group than in the control group, but there was no significant difference between the painful and non-painful jaw side in the TMD patients. Discussion. The hypothesis that TMD patients with chronic unilateral orofacial pain preferentially attend to somatosensory information at the painful side of the jaw was not statistically supported, although lack of power could not be ruled out as a reason for this. The findings are discussed within recent 
theories of pain-related attention. 
1 Do patients with chronic unilateral orofacial pain due to a temporomandibular disorder show increased attending to somatosensory input at the painful side of the jaw?

3 Stefaan Van Damme ${ }^{\mathrm{a}}$, Charlotte Vanden Bulcke ${ }^{\mathrm{a}}$, Linda Van den Berghe ${ }^{\mathrm{b}}$, Louise Poppe ${ }^{\mathrm{a}, \mathrm{c}}$, and

5 a Department of Experimental-Clinical and Health Psychology, Ghent University, Belgium.

$6 \quad{ }^{b}$ Department of Dentistry, Ghent University Hospital, Belgium

$7 \quad{ }^{\mathrm{c}}$ Department of Movement and Sports Sciences, Ghent University, Belgium

$8{ }^{\mathrm{d}}$ Center for Pain Research, University of Bath, United Kingdom

9 Corresponding author:

10 Stefaan Van Damme

11 E-mail address: stefaan.vandamme@ugent.be 


\section{Abstract}

13 Background. Patients with chronic orofacial pain due to temporomandibular disorders (TMD)

14 display alterations in somatosensory processing at the jaw, such as amplified perception of tactile

15 stimuli, but the underlying mechanisms remain unclear. This study investigated one possible

16 explanation, namely hypervigilance, and tested if TMD patients with unilateral pain showed

17 increased attending to somatosensory input at the painful side of the jaw.

18 Methods. TMD patients with chronic unilateral orofacial pain $(\mathrm{n}=20)$ and matched healthy

19 volunteers $(n=20)$ performed a temporal order judgment $($ TOJ $)$ task indicated which one of two

20 tactile stimuli, presented on each side of the jaw, they had perceived first. TOJ methodology

21 allows examining spatial bias in somatosensory processing speed. Furthermore, after each block

22 of trials, the participants rated the perceived intensity of tactile stimuli separately for both sides of

23 the jaw. Finally, questionnaires assessing pain catastrophizing, fear-avoidance beliefs, and pain

24 vigilance, were completed.

25 Results. TMD patients tended to perceive tactile stimuli at the painful jaw side as occurring

26 earlier in time than stimuli at the non-painful side but this effect did not reach conventional levels

27 of significance $(p=.07)$. In the control group, tactile stimuli were perceived as occurring

28 simultaneously. Secondary analyses indicated that the magnitude of spatial bias in the TMD

29 group is positively associated with the extent of fear-avoidance beliefs. Overall, intensity ratings

30 of tactile stimuli were significantly higher in the TMD group than in the control group, but there

31 was no significant difference between the painful and non-painful jaw side in the TMD patients.

32 Discussion. The hypothesis that TMD patients with chronic unilateral orofacial pain

33 preferentially attend to somatosensory information at the painful side of the jaw was not

34 statistically supported, although lack of power could not be ruled out as a reason for this. The

35 findings are discussed within recent theories of pain-related attention. 


\section{Introduction}

$$
\text { Temporomandibular disorders (TMD) represent a group of highly prevalent and disabling }
$$
conditions characterized by problems and dysfunctions in the jaw muscles and/or in the temporomandibular joint, with chronic orofacial pain as one of the typical features (Turner et al., 2001). TMD are poorly understood and difficult to treat, and likely to be affected by multiple factors (Suvinen et al., 2005). It has been proposed that abnormalities in somatosensory processing may play a role (Nebel et al., 2010). For instance, it has been found that TMD patients, relative to healthy controls, report tactile stimuli at the jaw as more intense (Ayesh et al., 2007) and display enhanced cortical responses to such stimuli (Alonso et al., 2010). However, insight in the exact nature and underlying mechanisms of somatosensory amplification in TMD is largely missing. For example, although TMD-related orofacial pain is more often unilateral than bilateral, studies investigating somatosensory amplification have rarely differentiated between the painful and non-painful side of the jaw (Ayesh et al., 2007; Kothari et al., 2015).

One possible source of amplified somatosensory perception in chronic pain patients is hypervigilance, that is, the process of habitually attending to pain-related information such as bodily cues (Chapman, 1978; Crombez et al., 2005; Hollins et al., 2009; McDermid et al., 1996). Hypervigilance is thought to emerge as a result of the experience of threat (Eccleston \& Crombez, 1999), for instance, in patients with high levels of pain-related fear (Vlaeyen \& Linton, 2000). It has been proposed that an important feature of hypervigilance is increased attending to somatosensory input at pain-relevant body locations (Van Damme et al., 2016). This idea has been extensively investigated in a series of studies in healthy volunteers indicating that experimentally induced threat of pain on one specific location of the body resulted in increased attending to somatosensory stimuli at that body location relative to other areas (Crombez et al., 1998; Durnez \& Van Damme, 2015; Vanden Bulcke et al., 2013, 2014, 2015; Van Hulle et al., 2015; but see Durnez \& Van Damme, 2016, for a failed replication). Applied to unilateral TMD 
61 pain, one would expect enhanced attending to somatosensory information specifically at the

62 painful side of the jaw. However, studies investigating location-specific attention biases in

63 clinical samples, including TMD, are currently lacking. Understanding the presence and nature of

64 somatosensory hypervigilance in TMD may help us determine new treatment for these patients.

65 The present study aimed to investigate whether TMD patients with unilateral orofacial pain

66 show a bias in somatosensory attending to the painful jaw side. For this purpose, we used a

67 Temporal Order Judgment (TOJ) paradigm (Piéron, 1952), assessing the order in which

68 individuals perceive pairs of stimuli. The paradigm has been typically used to investigate the law

69 of prior entry (Titchener, 1908), stating that attended stimuli come into consciousness earlier than

70 unattended stimuli (see Shore et al., 2005; Spence et al., 2001). More recently, TOJ tasks with

71 tactile stimuli have been used to demonstrate biases in somatosensory attention to or away from

72 pain-related body locations (Moseley et al., 2009; Reid et al., 2016; Van Damme et al., 2009;

73 Vanden Bulcke et al., 2013). In the current study, a sample of TMD patients with unilateral

74 chronic orofacial pain and a healthy control sample indicated which one of two tactile stimuli,

75 presented at either side of the jaw, they had perceived first. As the primary hypothesis, we tested

76 if TMD patients would be preferentially attending to the painful side of their jaw, which should

77 be reflected by a tendency to perceive tactile stimuli at the painful jaw side as occurring earlier

78 than tactile stimuli at the non-painful jaw side. No difference in tactile processing between both

79 sides of the jaw was expected in the control group. In addition, we conducted secondary analyses

80 in the TMD sample to examine correlations between spatial bias in tactile processing and a range

81 of self-reported pain cognitions, including pain-related fear, catastrophizing, and vigilance. 


\section{Materials \& Methods}

\section{Participants}

84 TMD patients were a convenience sample recruited in the Department of Dentistry at the

85 Ghent University Hospital. Inclusion criteria included having chronic (longer than 3 months)

86 unilateral orofacial pain due to a temporomandibular disorder assessed by the dentist, having an

87 age between 18 and 65 years, and being Dutch speaking. Exclusion criteria were the presence of

88 a severe psychiatric/neurological condition, and the presence of non-TMD related chronic pain

89 problems. Based upon power calculations (see further), we recruited 20 TMD patients and 20

90 healthy controls. Potential participants were informed about the possibility of participating by

91 means of a flyer and information given by the clinician. When they agreed to participate, they

92 received a phone call from the researcher providing more detailed information about the study.

93 Twenty-one patients agreed to participate in the experiment. It was not registered if they had

94 participated in previous studies. One woman (40 years, right-handed) had to be excluded because

95 she reported chronic widespread pain (fibromyalgia). The age of the remaining 20 TMD patients

96 (17 females) was 36.8 years $(\mathrm{SD}=11.6$, range $=22-59$ years $)$. Mean duration of pain was about

9714 months $(\mathrm{SD}=11.3$ months, range 4-36). Ten patients reported pain at the left jaw, the other

98 ten had pain at the right jaw. The majority of the sample $(70 \%)$ had not received treatment for

99 their TMD at the time of testing. An overview of the demographic characteristics is provided in

100 Table 1.

101 The control group was recruited from an existing database, consisting of individuals from the

102 general population who had expressed interest to participate in scientific studies of the Ghent

103 Health Psychology Research Group, by registering at a website

104 (http://www.healthpsychology.ugent.be/vrijwilligers). Potential participants were selected with

105 the aim of obtaining a control group matched at group level with the TMD group for age, gender

106 and educational level. Inclusion criteria for the control participants were being Dutch speaking 
107 and having an age between 18 and 65 years. Exclusion criteria were presence of chronic pain

108 complaints, presence of current orofacial pain, and presence of neurological/psychiatric

109 conditions. Eligible participants were contacted and provided with information about the study.

110 Twenty-one healthy volunteers were willing to participate. One man (23 years, right-handed) was

111 excluded for further analysis due to not attaining the requested performance criteria during the

112 task (see TOJ data handling). The age of the remaining 20 participants (18 females and 2 males)

113 was 36.9 years (range 20-63 years; $S D=13.9$ ). An overview of the demographic characteristics is

114 provided in Table 1. Statistical analyses showed no differences between groups in gender, $\chi^{2}(1)=$

$1150.23, p=.633$, mean age, $t(38)=-0.03, p=.980$, and educational level, $\chi^{2}(3)=0.69, p=.877$.

116 The study was approved by the Medical Ethical Committee of the Ghent University Hospital

117 (B670201213538). At the end of the experiment, all participants received 25 Euro as

118 reimbursement for their expenses. The experimental session lasted for approximately 1 hour and 119 a half.

Insert Table 1 about here

\section{Apparatus and materials}

122 Our tactile stimulation procedure was similar as what has been described in previous studies

123 (e.g., Vanden Bulcke et al., 2013). We presented tactile stimuli at both sides of the jaw with a

124 duration of $10 \mathrm{~ms}$ duration and a frequency of $200 \mathrm{~Hz}$, using two resonant-type tactors (C-2

125 TACTOR, Engineering Acoustics, Inc., Florida, http://www.eaiinfo.com/) consisting of a housing 126 of $3.05 \mathrm{~cm}$ diameter and $0.79 \mathrm{~cm}$ high, and a skin contactor of $0.76 \mathrm{~cm}$ diameter. In order to

127 control for differences in tactile sensitivity between both sides of the jaw, subjective stimulus

128 intensity was individually calibrated and matched between jaw sides before the experiment using

129 a double random staircase procedure (see Levitt, 1971; Weinstein, 1968). In a first phase, 
130 participants rated 24 stimuli presented on the left side of the jaw relative to a reference stimulus,

131 which was defined as the maximum intensity (power $=0.21$ Watt) on a 5-point Likert scale

132 ranging from 1 ('no sensation') to 5 ('maximum intensity'). The intensity that elicited an

133 averaged rating of 3 was used as the stimulus intensity for the left jaw side, and was the reference

134 stimulus for the second phase. In the second phase, participants rated 24 stimuli on the right jaw

135 side relative to the reference stimulus on the left jaw side on a 5-point Likert scale $(1=$ 'much

136 weaker', 2= 'weaker', 3= 'equally strong', 4= 'stronger', 5= 'much stronger'). The intensity that

137 elicited an averaged rating of 3 was used as the intensity of the stimulus at the right jaw side.

138 The task was programmed and controlled by the INQUISIT Millisecond software package

139 (Inquisit 3.0, Millisecond Software LLC, Seattle, WA, http://www.millisecond.com/) on a laptop

140 (Dell Vostro 3550).

141 TOJ paradigm

142 The tactile TOJ paradigm is considered a particularly suitable methodology to assess spatial

143 biases in tactile processing (Shore et al., 2005), and has also been used in the context of pain

144 (e.g., Moseley et al., 2009; Vanden Bulcke et al., 2014; but see Filbrich et al., 2016 and Van

145 Damme et al., 2016, for some methodological considerations and challenges). Specifically, two

146 tactile stimuli were administered, one on either side of the jaw, separated by one of 10 randomly

147 assigned stimulus onset asynchronies (SOAs) between -200 to $+200 \mathrm{~ms}(-200,-90,-55,-30,-10$,

$148+10,+30,+55,+60,+200 \mathrm{~ms})$. Each trial was preceded by the presentation of a fixation cross

$149(2000 \mathrm{~ms})$ in the middle of the screen. The participants reported verbally at which side of the jaw

150 they first had perceived the tactile stimulus. The experimenter registered their responses using a

151 keyboard. Note that the SOAs in this study were modified (made larger) as compared with

152 previous studies with undergraduate students $(-120,-60,-30,-15,-5,+5,+15,+30,+60,+120$;

153 see Vanden Bulcke et al., 2013), because pilot testing with TMD patients and adults from the 
154 general population indicated that with these values too many participants would not have been

155 able to meet the required performance criterion.

156 Self-report measures

157 A general questionnaire was used to collect sociodemographic data (gender, age, education

158 level, profession, family situation), presence of medical/psychological problems, and use of pain

159 medication. In the TMD group, this questionnaire also assessed which side of the jaw was

160 painful, duration of the TMD problem, current treatment status (whether they had received

161 treatment for their TMD or not), and presence of other chronic pain complaints. Several validated

162 self-report questionnaires measuring pain and pain-related cognitions were used. Internal

163 consistency (Cronbach's alpha) was acceptable for all these instruments (see Table 2).

164 Pain severity and pain interference were assessed by means of the Dutch version of the

165 Multidimensional Pain Inventory (MPI-DV; Kerns, Turk \& Rudy, 1985). This questionnaire

166 consists of 28 items rated on a 7-point scale measuring severity of the pain problem (e.g. 'Rate

167 the level of your pain at the present moment'), interference with daily-life activities (e.g. 'In

168 general, how much does your pain interfere with your day-to-day activities?'), perceived control

169 (e.g. 'During the past week how much control do you feel you have had over your life?'),

170 affective anxiety (e.g. 'During the past week how irritable have you been?') and social support

171 (e.g. 'How supportive or helpful is your significant other to you in your relation to your pain?').

172 Only the first two subscales were included.

173 The Tampa Scale for Kinesiophobia for Temporomandibular Disorders (TSK-TMD; Visscher

174 et al., 2010) consists of 12 items that need to be rated on a 4-point numerical rating scale $[1=$

175 "strongly disagree", 4 = "strongly agree"]. The subscale 'Activity Avoidance' (e.g. 'I am afraid

176 that I might injure myself if I move my jaw') consists of 7 items, whereas the other subscale

177 'Somatic focus' (e.g. 'My jaw is telling me that something is seriously wrong with it') consists of

1785 items. The TSK-TMD has been shown to be valid and reliable (Visscher et al., 2010). 
179 The Pain Catastrophizing Scale (PCS; Sullivan, Bisschop \& Pivik, 1995) is a 13-item scale to

180 assess catastrophic thoughts about pain in both non-clinical and clinical populations. Participants

181 are asked to reflect on past painful experiences and to indicate the degree to which they

182 experienced each of the 13 thoughts or feelings during pain on a 5-point scale from 0 (not at all)

183 to 4 (all the time). The PCS consists of 3 subscales: rumination (e.g., 'I keep thinking about how

184 much it hurts'), magnification (e.g., 'I become afraid that the pain may get worse'), and

185 helplessness (e.g., 'There's nothing I can do to reduce the intensity of the pain'). The Dutch

186 version of the PCS has been shown to be valid and reliable in both healthy populations and

187 chronic pain patients (Van Damme et al., 2002).

188 The Pain Vigilance and Awareness Questionnaire (PVAQ; McCracken, 1997) contains 16

189 items rated on a 6-point scale measuring self-reported vigilance for pain sensations (1= "never",

$1905=$ "always"). The PVAQ consists of two subscales: attention to pain (e.g., 'I focus on sensations

191 of pain'), and attention to changes in pain (e.g., 'I am quickly to notice changes in pain intensity').

192 The Dutch version of the PVAQ has been shown to be valid and reliable in both healthy

193 populations and chronic pain patients (Roelofs et al., 2002, 2003).

194 After each test phase, the participants were asked to rate the perceived intensity of the tactile

195 stimuli ('How intense did you experience the stimuli on your left/right jaw?') on an eleven-point

196 numerical rating scale (anchored $0=$ not at all and $10=$ very strongly). This was included to

197 check for potential sensitization or habituation effects.

198 Procedure

199 Upon arrival at the laboratory, participants were informed about the experimental procedures 200 and provided written informed consent. After this, they completed a series of questionnaires (see 201 self-report measures) in the following order: general questionnaire, MPI-DV, TSK-TMD (only

202 TMD patients), PCS, and PVAQ. Next, they were seated in front of the experimental apparatus.

203 Their forearms were positioned symmetrically on the table. The tactors were placed in the middle 
204 of the superficial head of the masseter muscle of each side of the jaw. The participants were

205 informed that they had to decide on each trial which stimulus had been presented first. The

206 accuracy of participants' responses was emphasized, rather than the speed. Participants wore

207 noise-cancelling headphones (PXC 350 Sennheiser) to prevent any interference from

208 environment noise. Following this, the session began with a practice block of twenty trials $(2$

209 trials per SOA). Next, four blocks of 70 trials (7 trials per SOA) were presented. The participants

210 were not given any feedback about their performance. After each block, they were asked to rate

211 perceived intensity of tactile stimuli at both jaws. These data were not used to adapt stimulus

212 intensity in case of left-right differences. The participants also completed several single rating

213 scales gauging concentration, effort, attention, and fatigue (not reported).

214 Data-analyses

215 TOJ data handling. The analyses were based upon procedures that have been commonly

216 described in the literature (Shore et al., 2005; Spence et al., 2001). The proportions of 'left-jaw-

217 first' and 'right-jaw-first' responses for all trials at each SOA, were converted into the

218 corresponding z-scores using a standardized cumulative normal distribution. The best-fitting

219 straight line was computed for each participant and the derived slope and intercept values were

220 used to compute the just noticeable difference (JND) and the point of subjective simultaneity

221 (PSS). JND is monotonically related to the slope of the psychometric function and indicates the

222 (virtual) interval needed to achieve 75\% correct performance, and as such provides a

223 standardized measure of the sensitivity of participants' temporal perception.

224 PSS refers to the (virtual) SOA at which participants report the two events (in this case: right

225 jaw first and left jaw first) equally often. The PSS is computed as the opposite of the intercept

226 divided by the slope from the best-fitting straight line. It is customary to code SOAs so that

227 negative values indicate that the test stimulus was presented before the reference stimulus. In the

228 TMD sample, we regard stimuli at the painful jaw side as test stimuli, while stimuli at the non- 
229 painful jaw side are labeled as reference stimuli. In the remainder of the manuscript, positive

230 SOAs refer to trials in which the stimulus at the non-painful jaw side preceded the stimulus at the

231 painful jaw side. When interpreting effects on the PSS measurement, it is thus important to keep

232 in mind that positive values indicate that stimuli stemming from the non-painful jaw side should

233 be presented before stimuli originating from the painful jaw side for both to be perceived as

234 simultaneously occurring. Correspondingly, positive PSS values indicate that tactile input at the

235 painful jaw side is prioritized over tactile input at the non-painful jaw side. In the control group,

236 we made the (arbitrary) choice to consider stimuli at the right jaw side as test stimuli, while

237 stimuli at the left jaw side were reference stimuli. Here, positive values indicate that stimuli

238 stemming from the left jaw side should be presented before stimuli originating from the right jaw

239 side for both to be perceived as simultaneously occurring. Correspondingly, positive PSS values

240 indicate that tactile input at the right jaw side is prioritized over tactile input at the left jaw side.

241 In line with Spence et al. (2001), we excluded participants (from both groups) from statistical

242 analysis when any of their PSS values was larger than the highest SOA ( $\pm 200 \mathrm{~ms})$. Participants

243 were also excluded when less than $80 \%$ of their responses on trials with the largest SOA $( \pm 200$

$244 \mathrm{~ms}$ ) was incorrect. Note that Spence et al. (2001) originally used a cut-off of $90 \%$, but that we

245 have previously decided to deviate from this because much more participants would have to be

246 excluded than what has been typically reported by Spence et al. (2001), even in undergraduate

247 students (see Vanden Bulcke et al., 2013, 2014). As a result, one participant of the control group

248 (male, right-handed) was removed from data analysis.

249 TOJ hypothesis testing. We tested in each group whether the obtained mean PSS value was

250 significantly different from the actual point of simultaneity (virtual SOA of $0 \mathrm{~ms}$ ) using one-

251 sample $t$-tests. Based upon the study by Vanden Bulcke et al. (2013), reporting an effect size of

2520.70 for enhanced tactile processing at a hand threatened by experimental pain (relative to the

253 other hand) in healthy volunteers, the current study was powered to detect an effect size of 0.70 
254 with $80 \%$ power at alpha $<.05$ for a one-sample $t$-test. As a result, at least 19 patients were

255 needed. As an additional analysis, we compared the mean PSS between the TMD group and the 256 control group. However, it should be noted that the meaning of these values is different for these

257 groups (see TOJ data handling). In the control group a positive PSS simply means that tactile

258 input at the right jaw side is prioritized over tactile input at the left jaw side. In the TMD sample a

259 positive PSS means that tactile input at the painful jaw side (irrespective of whether this is left or

260 right) is prioritized over tactile input at the non-painful jaw side. As a secondary, exploratory,

261 analysis, we tested within the TMD group whether the PSS was different between those that had

262 pain at the left jaw side $(N=10)$ and those that had pain at the right jaw side $(N=10)$, using an

263 independent-samples $t$-test. We had no specific hypotheses regarding the JND.

264 Perceived intensity of tactile stimuli. As a validity check, to prevent explanations of TOJ

265 effects in terms of differences in subjective tactile intensity, we compared perceived intensity

266 (average across the 4 blocks) between the painful and the non-painful side of the jaw in the TMD

267 group, and between the left and the right side of the jaw in the control group, using paired-

268 samples $t$-tests. As an exploratory analysis, we also compared average perceived tactile intensity

269 between the TMD and the control groups, using an independent samples $t$-test.

270 Questionnaires. For the TSK-TMD we only provide descriptive statistics for the TMD group.

271 For all other questionnaires (MPI, PCS, PVAQ) we compared scores between TMD patients and

272 controls, using independent-samples $t$-tests. As a secondary analysis, we calculated Pearson

273 correlations in the TMD group only, to examine possible associations between questionnaire

274 scores and the behavior measure of spatial bias (PSS).

275 In all analyses significance level was set at $p<.05$. For ease of comparison with the norms of

276 Cohen (1988), we calculated effect sizes for independent samples using the formula of Dunlap et

277 al. (1996). We determined whether Cohen's d was small $(<=0.20)$, medium $(<=0.50)$, or large

$278(<=0.80)$. We also report the $95 \%$ confidence intervals $(95 \% \mathrm{CI})$ of the effect sizes. 


\section{Results}

280 Sample characteristics

281 Table 2 represents the average scores and standard deviations for the self-report questionnaires

282 in the TMD group and the control group (except for TSK-TMD). Scores on the MPI were quite

283 similar as those found in a large $(N=491)$ sample of TMD patients (Reissman et al., 2008),

284 except for the pain severity subscale, which was almost 1 standard deviation lower in the current

285 sample. The mean score for the TSK-TMD $(M=24.75, S D=6.44$, range 14-40) was similar as

286 the score obtained in a large sample of TMD patients $(N=301, M=24.2, S D=6.9)$ (Visscher et

287 al., 2010).

288 With regard to the MPI, independent samples $t$-tests revealed that the TMD group had

289 significantly higher scores as compared to the control group (pain severity: $t(38)=3.33, p=.002$;

290 pain interference: $t(38)=2.67, p=.011)$. For the PVAQ, independent samples $t$-tests revealed

291 that the TMD group had significantly higher scores as compared to the control group, $t(38)=$

292 2.62, $p=.01$ ). No significant differences between both groups were found on the Pain

293 Catastrophizing Scale $(t(38)=0.55, p=0.58)$.

Tactile intensity and validity check

296 No significant difference in perceived intensity between tactile stimuli presented on the 297 painful side $(M=5.56, S D=2.02)$ and tactile stimuli presented on the non-painful side $(M=$ $2985.31, S D=2.27$ ) was found in TMD patients, $t(19)=0.80, p=0.44$. Perceived intensity of tactile 299 stimuli (across sides and blocks) was significantly higher in the TMD group $(M=5.44, S D=$

300 2.03) than in the control group $(M=3.98, S D=1.99), t(38)=2.29, p=.028(d=0.71[95 \% \mathrm{CI}$

$3010.07-1.35])$. When considering the results on perceived tactile intensity it is important to note 
302 that the actual intensity of administered tactile stimuli was not different between the TMD group $303(M=22.78, S D=3.47)$ and the control group $(M=21.93, S D=3.10), t(38)=0.82, p=.419$, and

304 that in the TMD group there was no significant difference in actual tactile intensity between the 305 painful $(M=23.05, S D=4.85)$ and the non-painful side of the jaw $(M=22.50, S D=3.86), t(19)$

$306=0.46, p=0.651$. Possible TOJ effects in TMD patients thus could not be due to differences in

307 tactile stimulation between the painful and non-painful side of the jaw.

308 Hypothesis testing

309 Analysis of the PSS values in the TMD group showed that tactile stimuli delivered to the non310 painful side of the jaw had to be delivered before tactile stimuli at the painful side, for both

311 stimuli to be perceived as occurring at the same time $(M=17.59, S D=41.51)$, but the mean

312 difference with the actual point of simultaneity $(0 \mathrm{~ms})$ was not significant, $t(19)=1.90, p=.07(d$

$313=0.42,[95 \%$ CI: $-0.19,1.03])$. There was no significant difference in PSS values between

314 patients with left jaw pain $(M=7.15, S D=41.89)$ and patients with right jaw pain $(M=28.02$,

$315 S D=40.51), t(18)=1.13, p=.27$. In the control group, the PSS was close to zero $(M=-0.12, S D$

$316=27.40$ ), and did not differ from the actual point of simultaneity (zero), $t(19)=-0.02, p=0.98$.

317 Note that the mean PSS was not significantly larger in the TMD group than in the healthy control

318 group, despite a medium effect size, $t(38)=1.59, p=0.12, d=0.50,95 \%$ CI $[-0.13,1.13]$.

319 The JND (about which no hypothesis was formulated) was not significantly different between 320 the TMD group $(M=73.87, S D=20.84)$ and the control group $(M=64.42, S D=15.42), t(38)=$ $3211.63, p=.11$.

322 Secondary analyses

323 Pearson Correlations in the TMD group are shown in Table 3. Of the 10 calculated correlations 324 between the PSS and the questionnaire scores, most did not reach significance, except for 1 325 significant positive correlation between the PSS and the somatic focus subscale of the TSK-TMD 
$326(r=.50, p=.025)$, suggesting that higher scores on this scale were associated with a tactile

327 processing bias toward the painful side of the jaw.

\section{Discussion}

330 This study investigated hypervigilance for somatosensory information in TMD patients with 331 chronic unilateral orofacial pain. Specifically, we tested the hypothesis that processing of tactile

332 stimuli would be biased towards the painful side of the jaw in TMD patients. While the average

333 PSS in healthy controls was approximately zero, it was positive (about $18 \mathrm{~ms}$ ) in the TMD group,

334 but the difference with the actual point of simultaneity $(0 \mathrm{~ms})$ did not reach conventional levels of

335 statistical significance $(p=.07)$, and there was also no significant difference with the control

336 group. Strong interpretation of these results is difficult, given that confidence intervals were

337 large, and that the study was only sufficiently powered to detect a medium effect size. It is

338 possible that true effects are smaller than what was expected based on the study of Vanden

339 Bulcke et al. (2013) because of several differences (left-right side of the jaw versus left-right

340 hand; natural tonic pain versus experimentally induced anticipation of phasic pain stimuli). A

341 follow-up study powered to detect small-to-medium effects with $90 \%$ chance is recommended

342 before drawing strong conclusions.

343 Exploratory correlation analyses were conducted to gain insight in individual variability of

344 spatial bias to the painful side of the jaw (i.e., PSS) in the TMD group. Most of the correlations

345 between PSS and self-reported cognitive-affective variables were not significant, but we

346 observed a significant positive association between the PSS and the "somatic focus" subscale of

347 the TSK-TMD, suggesting that those who tend to appraise somatic sensations in their jaw in a

348 threatening way, show a stronger spatial bias in tactile processing to the painful side of the jaw. 
349 This finding fits well with previous findings showing that levels of pain-related fear are

350 associated with self-reported jaw activity limitations and disability in TMD patients (Turner et al.,

351 2001; Visscher et al., 2010) and faster detection of innocuous electrical stimuli in chronic low

352 back pain patients (Peters et al., 2002). However, the importance of this significant correlation

353 should not be overstated, given the exploratory nature of this analysis, the small sample, and the

354 large amount of correlations increasing the risk of type-I error.

355 It is interesting to compare our results with previous findings in patients with other unilateral

356 pain complaints. Studies using tactile TOJ methodology have shown that in patients with both

357 unilateral chronic low back pain and complex regional pain syndrome, tactile processing at the

358 painful side appeared be attenuated (Moseley et al., 2009, 2012a, 2012b). More specific, these

359 authors found shifts in PSS so that tactile stimuli at the affected side had to be presented before

360 stimuli at the other side, to be perceived as simultaneous. It has been argued that impaired tactile

361 processing at the affected body part may be a consequence of disrupted spatial representation

362 (Moseley et al., 2009) or spatial "inattention" (Reid et al., 2016). Perhaps this feature is only

363 present in specific populations of chronic pain patients, and not in (our sample of) patients with

364 TMD. It should also be noted that there are minor methodological differences between our study

365 and other experiments, especially regarding the SOAs used in the tactile TOJ. More specifically,

366 we used SOAs between 10 and $200 \mathrm{~ms}$, whereas in the studies of Moseley and colleagues, the

367 range was between 10 and $240 \mathrm{~ms}$. It is unlikely, though, that these small differences would be

368 responsible for obtaining opposite effects. Nevertheless, our findings show that we should be

369 cautious in generalizing the idea of disrupted tactile processing in painful body parts to the entire

370 population of unilateral chronic pain patients (Van Damme et al., 2016). More research with more

371 diverse and larger samples of chronic pain patients (e.g., Van Damme et al., 2014, 2015),

372 allowing identification of moderating factors of enhanced versus attenuated tactile processing, is

373 highly recommended. 
374 Several issues deserve further discussion. First, the overall perceived intensity of tactile

375 stimuli (across both sides) was higher in the TMD group than in the control group. This is an

376 intriguing finding, because the objective (actual) intensity was not different between groups, and

377 it was individually calibrated to the same rating (3 on a 1-5 rating scale) before the start of the

378 experiment. This may suggest some form of sensitization or delayed habituation to

379 somatosensory input in TMD patients. Note that this is not in line with previous findings of

380 elevated tactile thresholds in these patients (Hollins \& Sigurdsson, 1998; Hollins et al., 1996).

381 Because amplified perception in the current study was not specific to the painful side, it cannot

382 account for any spatial attention bias. Also note that in the TMD group there was no difference in

383 tactile intensity between the painful and the non-painful side of the jaw, which is in line with

384 previous findings (Ayesh et al., 2015; Kothari et al., 2015), although these studies did not

385 exclusively include patients with unilateral pain, and also examined differences between the most

386 and the least painful side of the jaw in patients with bilateral pain.

387 Second, the spatial bias in TMD patients was small, and individual variability was high, with

388 secondary analyses suggesting stronger bias in those patients characterized by threatening

389 appraisal of bodily signals in the jaw. An interesting avenue for future research would be to

390 investigate the effect of contextual factors, such as anticipation of pain, on spatial bias. It should

391 be noted that in this study, there was no induction of any bodily threat. It might be that situations

392 in which patients actively anticipate pain would increase spatial bias to the painful jaw. It could

393 be recommended that future studies examine somatosensory processing while patients are

394 anticipating a painful dental procedure, or after experimentally inducing pain anticipation by

395 requiring patients to perform certain activities using the jaw muscles. There have been successful

396 attempts to implement pain-related physical activities in somatosensory attention paradigms in

397 healthy volunteers (Clauwaert et al., in press) as well as in whiplash patients with chronic neck

398 pain (Vangronsveld et al., 2007). Third, we should be cautious in generalizing the results to TMD 
399 patients with bilateral orofacial pain, and call for further research in these samples. Fourth, it has

400 to be noted that the study protocol and statistical plan have not been pre-registered for peer

401 review.

\section{Conclusions}

403 Our main hypothesis, that TMD patients would be characterized by a spatial tactile bias

404 towards the painful side of the jaw, was not statistically confirmed, although the effect was in the 405 expected direction. Given the higher than anticipated variability in PSS, inadequate power could 406 not be ruled out a possible reason for this lack of statistical significance. In addition, the

407 significant positive correlation between the amount of spatial bias and the somatic focus subscale 408 of the TSK-TMD, suggests that somatosensory attending to the painful side of the jaw might be

409 elevated especially in patients who are characterized by fear-avoidance beliefs, but a more 410 adequately powered study would be needed to confirm this.

\section{Acknowledgements}

412 The authors would like to thank all personnel of the Unit Orofacial Pain and

413 Temporomandibular Dysfunctions at Ghent University Hospital for their assistance in recruitment 414 of patients.

\section{References}

416 Alonso AA, Koutlas IG, Leuthold AC, Lewis SM, Georgopoulos AP. 2010. Cortical processing of

417 facial tactile stimuli in temporomandibular disorder as revealed by

418 magnetoencephalography. Experimental Brain Research 204:33-45. 
419 Ayesh EE, Jensen TS, Svensson P. 2007. Hypersensitivity to mechanical and intra-articular 420 electrical stimuli in persons with painful temporomandibular joints. Journal of Dental $421 \quad$ Research 86:1187-1192.

422 Chapman CR. 1978. Pain: The perception of noxious events. In Sternbach RA, editor. The 423 psychology of pain. New York: Raven Press, pp. 169-202.

424 Cohen J. 1988. Statistical power analysis for the behavioral sciences. San Diego, CA: McGraw$425 \quad$ Hill.

426 Clauwaert A, Danneels L, Torta D, Van Damme S. 2017. Attentional modulations of somatosensory processing during pain-related movement preparation and execution.

429 Crombez G, Eccleston C, Baeyens F, Eelen P. 1998. When somatic information threatens, $430 \quad$ catastrophic thinking about pain enhances attentional interference. Pain 75:187-198.

431 Crombez G, Van Damme S, Eccleston C. 2005. Hypervigilance to pain: An experimental and $432 \quad$ clinical analysis. Pain 116:4-7.

433 Dunlap WP, Cortina JM, Vaslow JB, Burke MJ. 1996. Meta-analysis of experiments with matched groups or repeated measures designs. Psychological Methods 1:170-177

435 Durnez W, Van Damme S. 2015. Trying to fix a painful problem: The impact of pain control 436 attempts on the attentional prioritization of a threatened body location. Journal of Pain

438 Durnez W, Van Damme S. 2016. No evidence for threat-induced spatial prioritization of 439 somatosensory stimulation during pain control using a synchrony judgment paradigm. $440 \quad$ PLoS ONE 11 (6): e0156648.

441 Eccleston C, Crombez G. 1999. Pain demands attention: A cognitive-affective model on the 442 interruptive function of pain. Psychological Bulletin 125:356-366. 
443 Filbrich L, Torta DM, Vanderclausen C, Azanon E, Legrain V. 2016. Using temporal order

444 judgments to investigate attention bias tward pain and threat-related information:

445 Methodological and theoretical issues. Consciousness and Cognition 41:135-138.

446 Hollins M, Harper D, Gallagher S, Owings EW, Lim PF, Miller V, Siddiqqi MQ, Maixner W.

447 2009. Perceived intensity and unpleasantness of cutaneous and auditory stimuli: An

448 evaluation of the generalized hypervigilance hypothesis. Pain 141:215-221.

449 Hollins M, Sigurdsson A. 1998. Vibrotactile amplitude and frequency discrimination in 450 temporomandibular disorders. Pain 75:56-67.

451 Hollins M, Sigurdsson A, Fillingim L, Goble AK. 1996. Vibrotactile threshold is elevated in 452 temporomandibular disorders. Pain 67:89-96.

453 Kerns RD, Turk DC, Rudy TE. 1985. The West Haven-Yale Multidimensional Pain Inventory. $454 \quad$ Pain 23:345-356.

455 Kothari SF, Baad-Hansen L, Oono Y, Svensson P. 2015. Somatosensory assessment and 456 conditioned pain modulation in temporomandibular disorders pain patients. Pain 457 $156: 2545-55$.

458 Levitt H. 1971. Transformed up-down methods in psychoacoustics. Journal of Acoustical Society 459 of America 49:467-477.

460 McCracken L. 1997. Attention to pain in persons with chronic pain: a behavioral approach. $461 \quad$ Behavior Therapy 28:271-284.

462 McDermid AJ, Rollman GB, McCain GA. 1996. Generalized hypervigilance in fibromyalgia: 463 Evidence of perceptual amplification. Pain 66:133-144.

464 Moore DJ, Keogh E, Crombez G, Eccleston C. 2013. Methods for studying naturally occurring 465 human pain and their analogues. Pain 154:190-199. 
466 Moseley GL, Gallace A, Iannetti GD. 2012a. Spatially defined modulation of skin temperature

467 and hand ownership of both hands in patients with unilateral complex regional pain

$468 \quad$ syndrome. Brain 135:3676-3686.

469 Moseley GL, Gallace A, Spence C. 2009. Space-based, but not arm-based, shift in tactile

470 processing in complex regional pain syndrome and its relationship to cooling of the

$471 \quad$ affected limb. Brain 132:3142-3151.

472 Moseley GL, Gallagher L, Gallace A. 2012b. Neglect-like tactile dysfunction in chronic back $473 \quad$ pain. Neurology 79:327-332.

474 Nebel MB, Folger S, Tommerdahl M, Hollins M, McGlone F, Essick G. 2010.

475 Temporomandibular disorder modifies cortical response to tactile stimulation. Journal of $476 \quad$ Pain 11:1083-94.

477 Peters ML, Vlaeyen JWS, Kunnen AMW. 2002. Is pain-related fear a predictor of somatosensory 478 hypervigilance in chronic low back pain patients? Behaviour Research and Therapy $479 \quad 40: 85-103$.

480 Piéron H. 1952. The sensations: their functions, processes and mechanisms. New Haven: Yale $481 \quad$ University Press.

482 Reid E, Wallwork SB, Harvie D, Chalmers KJ, Gallace A, Spence C, Moseley GL. 2016. A new 483 kind of spatial inattention associated with chronic limb pain? Annals of Neurology $484 \quad 79: 701-704$.

485 Reissman DR, John MT, Wassell RW, Hinz A. 2008. Psychosocial profiles of diagnostic 486 subgroups of temporomandibular disorder patients. European Journal of Orofacial $487 \quad$ Science 116:237-244.

488 Roelofs J, Peters ML, McCracken L, Vlaeyen JWS. 2003. The Pain Vigilance and Awareness 489 Questionnaire (PVAQ): Further psychometric evaluation in fibromyalgia and other $490 \quad$ chronic pain syndromes. Pain 101:299-306. 
491 Roelofs J, Peters M, Muris P, Vlaeyen JWS. 2002. Dutch version of the Pain Vigilance and

492

493

494

495

496

497

498

499

500

501

502

503

504

505

506

507

508

509

510

511

512

513

514

Awareness Questionnaire: validity and reliability in a pain-free population. Behaviour Research and Therapy 40:1081-1090.

Shore D, Gray K, Spry E, Spence C. 2005. Spatial modulation of tactile temporal-order judgments. Perception 34:1251-1262.

Spence C, Shore D, Klein R. 2001. Multisensory prior entry. Journal of Experimental Psychology: General 130:799-832.

Sullivan M, Bischop S, Pivik J. 1995. The Pain Catastrophizing Scale: Development and validation. Psychological Assessment 7:524-532.

Suvinen TI, Reade PC, Kemppainen P, Könönen M, Dworkin SF. 2005. Review of aetiological concepts of temporomandibular pain disorders: towards a biopsychosocial model for integration of physical disorder factors with psychological and psychosocial illness impact factors. European Journal of Pain 9:613-633.

Titchener EB. 1908. Lectures on the elementary psychology of feeling and attention. New York: Macmillan.

Turner JA, Dworkin SF, Mancl L, Huggins KH, Truelove EL. 2001. The role of beliefs, catastrophizing, and coping in the functioning of patients with temporamandibular disorders. Pain 92:41-51.

Van Damme S, Crombez G, Bijttebier P, Goubert L, Van Houdenhove B. 2002. A confirmatory factor analysis of the Pain Catastrophizing Scale: invariant factor structure across clinical and non-clinical populations. Pain 96:319-324.

Van Damme S, Gallace A, Spence C, Crombez G, Moseley GL. 2009. Does the sight of physical threat induce a tactile processing bias? Modality-specific attentional facilitation induced by viewing threatening pictures. Brain Research 1253:100-106. 
515 Van Damme S, Legrain V, Vogt J, Crombez G. 2010. Keeping pain in mind: A motivational 516 account of attention to pain. Neuroscience and Biobehavioral Reviews 34:204-213.

517 Van Damme S, Vanden Bulcke C, Durnez W, Crombez G. 2016. Attentional bias to pain-relevant 518 body locations: new methods, new challenges. Consciousness and Cognition 43:128-132.

519 Van Damme S, Van Hulle L, Danneels L, Spence C, Crombez G. 2014. The effect of chronic low 520 back pain on tactile suppression during back movements. Human Movement Science $521 \quad 37: 87-100$.

522 Van Damme S, Van Hulle L, Spence C, Devulder J, Brusselmans G, Crombez G. 2015.

523 Hypervigilance for innocuous tactile stimuli in patients with fibromyalgia: an

524 experimental approach. European Journal of Pain 19:706-714.

525 Vanden Bulcke C, Crombez G, Durnez W, Van Damme S. 2015. Is attentional prioritization on a 526 location where pain is expected modality-specific or multisensory? Consciousness and 527 Cognition 36:246-255.

528 Vanden Bulcke C, Crombez G, Spence C, Van Damme S. 2014. Are the spatial features of bodily 529 threat limited to the exact location where pain is expected? Acta Psychologica 153:113$530 \quad 119$.

531 Vanden Bulcke C, Van Damme S, Durnez W, Crombez G. 2013. The anticipation of pain at a 532 specific location of the body prioritizes tactile stimuli at that location. Pain 154:1464$533 \quad 1468$.

534 Vangronsveld K, Van Damme S, Peters M., Vlaeyen JWS, Goossens M, Crombez G. 2007. An 535 experimental investigation on attentional interference by threatening neck movements in $536 \quad$ chronic whiplash patients. Pain 127:121-128.

537 Van Hulle L, Durnez W, Crombez G, Van Damme S. 2015. Detection of tactile change on a 538 bodily location where pain is expected. Perceptual and Motor Skills 120:1-13. 
539 Visscher CM, Ohrbach R, van Wijk AJ, Wilkosz M, Naeije M. 2010. The Tampa Scale for 540 Kinesiophobia for Temporomandibular Disorders (TSK-TMD). Pain 150:492-500.

541 Vlaeyen JWS, Linton SJ. 2000. Fear-avoidance and its consequences in chronic musculoskeletal 542 pain: A state of the art. Pain 85:317-332.

543 Weinstein S. 1968. Intensive and extensive aspects of tactile sensitivity as a function of body part, $544 \quad$ sex and laterality. Springfield: Thomas. 


\section{Table $\mathbf{1}$ (on next page)}

Demographic characteristics of the patient and control group 
Table 1

Demographic characteristics of the patient and control group

\begin{tabular}{|c|c|c|c|c|}
\hline & TMD patients & & Control group & \\
\hline & $\mathrm{M} \pm \mathrm{SD}$ & $\mathrm{N}(\%)$ & $\mathrm{M} \pm \mathrm{SD}$ & $\mathrm{N}(\%)$ \\
\hline Men & & $3(15 \%)$ & & $2(10 \%)$ \\
\hline Women & & $17(85 \%)$ & & $18(90 \%)$ \\
\hline Age (in years) & $36.8 \pm 11.66$ & & $36.9 \pm 13.90$ & \\
\hline & (range 22-59) & & (range 20-63) & \\
\hline \multicolumn{5}{|l|}{ Family situation } \\
\hline single & & $4(20 \%)$ & & $12(60 \%)$ \\
\hline living together & & $5(25 \%)$ & & $4(20 \%)$ \\
\hline married & & $8(40 \%)$ & & $4(20 \%)$ \\
\hline widow(er) & & $3(15 \%)$ & & 0 \\
\hline \multicolumn{5}{|l|}{ Educational level } \\
\hline primary education & & 0 & & 0 \\
\hline lower secondary education & & $2(10 \%)$ & & $1(5 \%)$ \\
\hline higher secondary education & & $6(30 \%)$ & & $8(40 \%)$ \\
\hline higher education & & $4(20 \%)$ & & $4(20 \%)$ \\
\hline higher education: university & & $8(40 \%)$ & & $7(35 \%)$ \\
\hline \multicolumn{5}{|l|}{ Profession } \\
\hline housemen/housewife & & $1(5 \%)$ & & $1(5 \%)$ \\
\hline laborer & & $2(10 \%)$ & & 0 \\
\hline employee & & $10(50 \%)$ & & $10(50 \%)$ \\
\hline professional & & 0 & & 0 \\
\hline senior manager & & 0 & & $1(5 \%)$ \\
\hline disabled & & $3(15 \%)$ & & $1(5 \%)$ \\
\hline student & & $4(20 \%)$ & & $5(25 \%)$ \\
\hline job seeker & & 0 & & $2(10 \%)$ \\
\hline
\end{tabular}




\section{Table 2 (on next page)}

Means (and standard deviations) and Cronbach's alpha of self-report questionnaires in both groups 
Table 2

Means (and standard deviations) and Cronbach's alpha of self-report questionnaires in both groups

\begin{tabular}{|c|c|c|c|c|}
\hline & \multicolumn{2}{|l|}{ TMD group } & \multicolumn{2}{|c|}{ Control group } \\
\hline & $\mathrm{M}(\mathrm{SD})$ & Alpha & $\mathrm{M}(\mathrm{SD})$ & Alpha \\
\hline MPI: pain severity & $1.9(1.1)$ & .70 & $0.9(0.9)$ & .69 \\
\hline MPI: interference & $2.0(1.9)$ & .96 & $0.8(1.0)$ & .95 \\
\hline TSK-TMD: total & $24.8(6.4)$ & .81 & - & - \\
\hline TSK-TMD: activity avoidance & $16.5(4.7)$ & .78 & - & - \\
\hline TSK-TMD: somatic focus & $8.3(2.6)$ & .63 & - & - \\
\hline PVAQ: total & $45.1(15.5)$ & .94 & $32.3(13.1)$ & .85 \\
\hline PVAQ: attention to pain & $26.0(9.8)$ & .91 & $16.3(7.4)$ & .77 \\
\hline PVAQ: attention to changes in pain & $19.1(6.8)$ & .88 & $16.1(8.9)$ & .94 \\
\hline PCS: total & $18.4(13.0)$ & .96 & $16.3(11.0)$ & .93 \\
\hline PCS: rumination & $6.7(4.2)$ & .86 & $6.9(4.2)$ & .90 \\
\hline PCS: magnification & $3.8(3.3)$ & .85 & $3.5(2.7)$ & .73 \\
\hline PCS: helplessness & $7.9(6.2)$ & .93 & $6.0(5.3)$ & .89 \\
\hline
\end{tabular}


Table 3(on next page)

Correlations between self-report measures and the TOJ outcome measure (PSS) for the TMD group 
Table 3

Correlations between self-report measures and the TOJ outcome measure (PSS) for the TMD group

\begin{tabular}{|c|c|c|c|c|c|c|c|c|c|c|}
\hline & 1 & 2 & 3 & 4 & 5 & 6 & 7 & 8 & 9 & 10 \\
\hline 1. PSS & - & & & & & & & & & \\
\hline 2. TSK-TMD (TOT) & .31 & - & & & & & & & & \\
\hline 3. TSK-TMD (AA) & .15 & $.94^{\mathrm{c}}$ & - & & & & & & & \\
\hline 4. TSK-TMD (SF) & $.50^{a}$ & $.78^{\mathrm{c}}$ & $.53^{\mathrm{a}}$ & - & & & & & & \\
\hline 5. PVAQ (TOT) & .17 & .29 & .10 & $.55^{\mathrm{a}}$ & - & & & & & \\
\hline 6. PVAQ (PAIN) & .16 & .27 & .05 & $.59^{\mathrm{b}}$ & $.95^{\mathrm{c}}$ & - & & & & \\
\hline 7. PVAQ (CHANGES) & .15 & .27 & .14 & .41 & $.90^{\mathrm{c}}$ & $.73^{\mathrm{c}}$ & - & & & \\
\hline 8. PCS (TOT) & .09 & .43 & $.45^{\mathrm{a}}$ & .26 & .40 & .37 & .38 & - & & \\
\hline 9. PCS (RUM) & -.13 & .27 & .31 & .10 & .44 & .42 & .39 & $.93^{\mathrm{c}}$ & - & \\
\hline 10. PCS (MAG) & .29 & $.50^{\mathrm{a}}$ & $.47^{\mathrm{a}}$ & .38 & .37 & .36 & .32 & $.93^{\mathrm{c}}$ & $.76^{\mathrm{c}}$ & - \\
\hline 11. PCS (HELP) & .11 & $.46^{\mathrm{a}}$ & $.48^{\mathrm{a}}$ & .27 & .35 & .31 & .36 & $.98^{\mathrm{c}}$ & $.87^{\mathrm{c}}$ & $.90^{\mathrm{c}}$ \\
\hline
\end{tabular}

Note: $\mathrm{TOT}=$ total score; $\mathrm{AA}=$ Activity Avoidance; $\mathrm{SF}=$ Somatic Focus; PAIN = Attention to pain; CHANGES = Attention to pain changes;

RUM = rumination; $M A G=$ magnification; HELP $=$ Helplessness 